\title{
Blood-brain barrier and cerebral blood flow: Age differences in hemorrhagic stroke
}

\author{
Semyachkina-Glushkovskaya Oxana, ${ }^{*},+\neq$, Sindeeva Olga*,, Abdurashitov Arkady ${ }^{\dagger, * *}$,

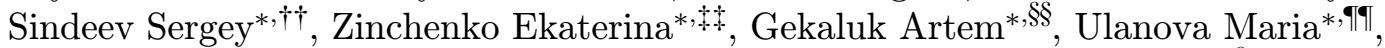 \\ Mohanad Kassim*,||l|, Yankovskaya Ludmilat*** and Tuchin Valery ${ }^{\dagger, \S, \dagger \dagger}$ \\ *Department of Physiology of Human and Animals \\ Saratov State University, Astrakhanskaya Str. 83 \\ Saratov 410012, Russia \\ ${ }^{\dagger}$ Department of Optics and Biophotonics \\ Saratov State University, Astrakhanskaya Str. 83 \\ Saratov 410012, Russia \\ *Department of Therapy, Grodno Medical State University \\ Limozha Str. 23, Grodno 230005, Byelorussia \\ ${ }^{\S}$ Laboratory of Biophotonics \\ Tomsk State University, Tomsk 634050, Russia \\ Tglushkovskaya@mail.ru \\ "mouse-oa@rambler.ru \\ **aarkady@icloud.com \\ †sbw@bk.ru \\ \#odonata1108@yandex.ru \\ \$gekalyuk.a@mail.ru \\ qT/maru-ulanova@mail.ru \\ IIIImohanadbio7r@yahoo.com \\ ***yankovliuda@yandex.ru \\ ††tuchin@mail.ru
}

Received 15 May 2015

Accepted 25 August 2015

Published 5 October 2015

\begin{abstract}
Neonatal stroke is similar to the stroke that occurs in adults and produces a significant morbidity and long-term neurologic and cognitive deficits. There are important differences in the factors, clinical events and outcomes associated with the stroke in infants and adults. However, mechanisms underlying age differences in the stroke development remain largely unknown. Therefore, treatment guidelines for neonatal stroke must extrapolate from the adult data that is
\end{abstract}

\$+Corresponding author.

This is an Open Access article published by World Scientific Publishing Company. It is distributed under the terms of the Creative Commons Attribution 3.0 (CC-BY) License. Further distribution of this work is permitted, provided the original work is properly cited. 


\section{S.-G. Oxana et al.}

often not suitable for children. The new information about differences between neonatal and adult stroke is essential for identification of significant areas for future treatment and effective prevention of neonatal stroke. Here, we studied the development of stress-induced hemorrhagic stroke and possible mechanisms underlying these processes in newborn and adult rats. Using histological methods and magnetic resonance imaging, we found age differences in the type of intracranial hemorrhages. Newborn rats demonstrated small superficial bleedings in the cortex while adult rats had more severe deep bleedings in the cerebellum. Using Doppler optical coherent tomography, we found higher stress-reactivity of the sagittal sinus to deleterious effects of stress in newborn vs. adult rats suggesting that the cerebral veins are more vulnerable to negative stress factors in neonatal vs. adult brain in rats. However, adult but not newborn rats demonstrated the stroke-induced breakdown of blood brain barrier (BBB) permeability. The one of possible mechanisms underlying the higher resistance to stress-related stroke injures of cerebral vessels in newborn rats compared with adult animals is the greater expression of two main tight junction proteins of $\mathrm{BBB}$ (occludin and claudin-5) in neonatal vs. mature brain in rats.

Keywords: Stroke; age differences; cerebral blood flow; brain blood barrier.

\section{Introduction}

The incidence of stroke in neonates is similar to that in the elderly. ${ }^{1}$ However, there are important differences in the factors associated with stroke in children when compared with adults. ${ }^{2,3}$ These differences likely play a role in the different outcomes, which tend to worsen with age. There are two types of strokes - hemorrhagic and ischemic. The hemorrhagic stroke accounts for between $39 \%$ to $54 \%$ of all neonatal strokes, that indicates that intracerebral and subarachnoid hemorrhage are important causes of childhood stroke. ${ }^{4-7}$ In contrast to neonates, hemorrhagic stroke in adults accounts for $6.5 \%$ to $13 \%$ of all strokes, ${ }^{8-10}$ while ischemic stroke accounts for almost $80 \%$ of the total cases of adult stroke. ${ }^{11}$ Thus, primary hemorrhage is an important component of neonatal but not adult stroke and is a greater source of mortality and morbidity in neonates than in adults.

The mechanism underlying age differences in the stroke is poorly understood but emerging evidence suggests that cerebral vascular abnormalities such as breakdown of the blood-brain barrier (BBB) and disturbances of the cerebral blood flow $(\mathrm{CBF})$ are an important contributing factors. ${ }^{12-14}$ The BBB's functions and gene expression of BBB endothelial proteins undergo significant changes in normal brain from embryonic development to adulthood. ${ }^{15-17}$ The CBF quantification is different in age groups both normal state and in patients with stroke. ${ }^{12}$ However, the studies of physiological processes responsible for age-related differences in the structure and functions of the BBB as well as in the $\mathrm{CBF}$ are extremely limited; our knowledge on the role of these processes in mechanisms of stroke comes from a number of experimental and clinical works on adults. Therefore, treatment guidelines for neonatal stroke must extrapolate from the adult data but the application of adult data in children is highly questionable. ${ }^{4,18-20}$ For better understanding of mechanisms underlying age differences in the stroke development, here we have studied the stroke-related changes in the BBB permeability and the $\mathrm{CBF}$ in newborn and adult rats using histological assay and magnetic resonance imaging of brain tissues, Doppler coherent tomography for monitoring of $\mathrm{CBF}$, color and adrenaline tests for analysis of the $\mathrm{BBB}$ penetration, wester blotting and immunochemistry for the study of expression of tight junction proteins of BBB.

\section{Methods and Materials}

\subsection{Subjects}

Experiments were carried out in newborn mongrel rats 2-3 days old and adult rats weighing $250-280 \mathrm{~g}$. Animals were divided into two groups: (1) the control group including intact, unstressed newborn rats $(n=11)$ and adult rats $(n=10),(2)$ the poststroke group (24h after stress-off) including newborn rats $(n=17)$ and adult rats $(n=15)$ with intracranial hemorrhages.

All procedures were performed in accordance with the "Guide for the Care and Use of Laboratory Animals.". ${ }^{21}$ The experimental protocols were 
approved by the Committee for the Care and Use of Laboratory Animals at Saratov State University (Saratov, Russia). The rats were housed at $25 \pm 2{ }^{\circ} \mathrm{C}, 55 \%$ humidity, $12: 12 \mathrm{~h}$ light/dark cycle, food and water ad libitum.

To induce hemorrhagic stroke, newborn and adult rats underwent severe sound stress $(17 \mathrm{~Hz}$, $120 \mathrm{~dB}$ ) during $2 \mathrm{~h}$. The details of performance of experiment modeling of hemorrhagic stroke is described here. ${ }^{22-25}$ This procedure was performed in the Plexiglas chamber (the volume is $2000 \mathrm{sm}^{3}$ ) absorbing and amplifying of deleterious effects of sound on the rats.

\subsection{CBF measuring}

The measurement of cerebral circulation was performed through the fontanel in anesthetized newborn rats (isoflurane - an inhalational anesthetic) with fixed head and scalp incision (the dura mater was left intact). Anesthetic depth was assessed by periodically monitoring the rear foot reflex.

In adult rats, the epicranium was exposed by a parieto-occipital midline skin incision. With the use of microsurgical technique, the periosteum was pushed back and biparietal parasagittal grooveshaped trephinations $(1.5 \times 4 \mathrm{~mm})$ was performed with a microdrill (Mikroton, Aesculap) during continuous irrigation with saline to prevent heating of the tissue. Special care was taken to prevent penetration of the dura mater.

Commercial swept source optical coherence tomography system OCS1300SS (Thorlabs Inc. USA) operating at $1325 \mathrm{~nm}$ central wavelength and $100 \mathrm{~nm}$ bandwidth was used for structural and Doppler imaging of cerebral vessels. The aperture angle of the output lens LSM03 mounted in OCS1300SS was 7.5 . Velocity of moving cells was calculated as

$$
V=\frac{\lambda_{0} f_{\mathrm{D}}}{2 n \cos \theta},
$$

where $\lambda_{0}$ is the central wavelength, $f_{\mathrm{D}}$ is the Doppler frequency shift, $n$ is the refractive index considered to be equal to 1.4 and $\theta$ is the angle between directions of incident radiation and cell movement. The Doppler frequency shift $f_{\mathrm{D}}$ was calculated as the rate of phase changes in the DOCT signal:

$$
f_{\mathrm{D}}=\frac{\Delta \varphi}{2 \pi} f_{\mathrm{A}} .
$$

Here $\Delta \varphi$ is the phase difference that can be retrieved from the phase maps produced by the Thorlabs software, $f_{\mathrm{A}}$ is the axial scan rate which is equal to $16 \mathrm{kHz}$. Since $\Delta \varphi$ is wrapped modulo $\pi$, in order to get actual values of $f_{\mathrm{D}}$, the standard unwrapping procedure is used.

\subsection{The evaluation of stress-induced brain injury}

To determine the incidence and topography of stroke in rats, we used T2-weighted magnetic resonance imaging (MRI, Hitachi Medical Corporation, Japan) and histological analysis of the brain tissue. For histological study, the animals were sacrificed at the end of the experiments, brain tissue samples were fixed in $10 \%$ formalin for $24 \mathrm{~h}$. The formalin fixed specimens were embedded in paraffin, sectioned $(3,5 \mu \mathrm{m})$ and stained with haematoxylin and eosin. The histological sections were evaluated by light microscopy.

\subsection{The study of BBB permeability}

To assess of BBB permeability, we used the color and pharmacological tests. The standard procedure to analyze of BBB permeability is Evans Blue injection and visualization. In the blood, this dye (961 Da) is becoming strongly bound to the albumin fraction of serum and makes a high molecular complex $(68.5 \mathrm{kDa})$ that does not permeate the intact BBB. ${ }^{26} 2 \%$ Evans Blue solution in $0.1 \mathrm{M}$ PBS was injected into the jugular vein $(0.2 \mathrm{ml} / 100 \mathrm{~g})$. $24 \mathrm{~h}$ after injection, the brain was carefully removed for visualization of blue color of brain tissues.

In our previous study, we found different effect of adrenaline on the $\mathrm{CBF}$ in newborn rats before and after stroke. ${ }^{25}$ Adrenaline caused vasoconstriction of cerebral vessels in healthy rats but did not induce any changes in the $\mathrm{CBF}$ in rats with intracranial hemorrhages, probably due to decrease in the $\mathrm{BBB}$ permeability. ${ }^{13}$ Here, we proposed adrenaline test as additional indirect method to the study of $\mathrm{BBB}$ permeability as well as to determine effectiveness of adrenaline as a main course in therapy of stroke $\mathrm{e}^{27-29}$ in neonates and adults.

The expression of tight junction proteins of the $\mathrm{BBB}$ was determined in lysates from the normal brain of newborn and adult rats using Western blot analysis and immunohistochemistry (mouse 


\section{S.-G. Oxana et al.}

Table 1. The frequency of stress-induced hemorrhages in the different anatomical structures of the brain in adult and full term newborn rats.

\begin{tabular}{|c|c|c|c|}
\hline \multirow{2}{*}{$\begin{array}{l}\text { Intracranial } \\
\text { hemorrhage type }\end{array}$} & \multirow[b]{2}{*}{ Definitions } & \multicolumn{2}{|c|}{ Comments } \\
\hline & & Adult rats $(n=15)$ & Newborn rats $(n=17)$ \\
\hline \multicolumn{4}{|c|}{ Hemorrhages outside of the brain } \\
\hline Epidural hemorrhage & $\begin{array}{l}\text { Blood between the skull and } \\
\text { the dura }\end{array}$ & - & - \\
\hline Subdural hemorrhage & $\begin{array}{l}\text { Blood between the dura and } \\
\text { arachnoid membrane }\end{array}$ & - & - \\
\hline Subarachnoid hemorrhage & $\begin{array}{l}\text { Blood between arachnoid } \\
\text { membrane and pia matter }\end{array}$ & - & - \\
\hline \multicolumn{4}{|l|}{ Intracerebral hemorrhages } \\
\hline $\begin{array}{l}\text { Cortical and subcortical } \\
\text { hemorrhage }\end{array}$ & $\begin{array}{l}\text { Blood within brain cortical } \\
\text { tissues }\end{array}$ & $\begin{array}{l}\text { Uncommon, this type of } \\
\text { hemorrhage was } \\
\text { accompanied by } \\
\text { cerebellar bleeding } \\
(n=1)\end{array}$ & $\begin{array}{l}\text { Common type, all rats } \\
\text { demonstrated small infarction } \\
\text { in the brain cortex (Fig. } 1 \\
\text { shows a typical example of this } \\
\text { type of bleeding) }\end{array}$ \\
\hline Cerebellar hemorrhage & Blood within cerebellum & $\begin{array}{l}\text { Most common type, } 14 \\
\text { rats demonstrated } \\
\text { cerebellar hemorrhages } \\
\text { (Fig. } 1 \text { shows a typical } \\
\text { example of this type of } \\
\text { bleeding) }\end{array}$ & - \\
\hline Intraventricular hemorrhage & $\begin{array}{c}\text { Blood in the brain ventricles } \\
\text { (lateral, third and fourth) }\end{array}$ & - & - \\
\hline
\end{tabular}

anti-occludin (1:500, Invitrogen), mouse anti-claudin-5 (1:500, Invitrogen)).

\subsection{Statistical analysis}

Results were reported as mean \pm standard error of the mean (SEM). Differences from the initial level in the same group were evaluated by the Wilcoxon test. Intergroup differences were evaluated using the Mann-Whitney test and ANOVA-2 (post hoc analysis with the Duncan's rank test). Significance levels were set at $p<0.05$ for all analyzes.

\section{Results}

In the first step of experiment, we studied age-related differences in development of hemorrhagic stroke in newborn and adult rats using histological and MRT analysis. The intracranial hemorrhages (induced by severe sound) occurred with the latent period $-24 \mathrm{~h}$ after stress independently of age. The frequency of different types of stress-induced hemorrhages in the brain of animals of both ages is presented in Table 1 . The adult and newborn rats demonstrated different types of hemorrhages in the different anatomical structures of the brain. Indeed, all newborn rats had small hemorrhages in the cortical and subcortical tissues. The adult rats were presented with hemorrhages in the cerebellum and cerebellar nucleus, we found only one case with the cortical bleeding that also was accompanied by cerebellar hemorrhage. Figure 1 shows a typical example of common type of stress-induced hemorrhages in newborn and adult rats. Histological study showed that the adult rats demonstrated more severe hemorrhagic brain injuries compared with newborn rats. The average size of brain bleeding was in newborn rats $0.004 \mathrm{~mm}^{2}$ while in adult rats is $-0.073 \mathrm{~mm}^{2}$. Figure 2 shows a typical example of size of brain hemorrhage in newborn and adult rats.

In the second step of our study, we analyzed the age differences in the stroke-related cerebral venous disturbances focusing on the changes in hemodynamic parameters of the sagittal sinus that is major venous sinus collecting blood from the small veins of the brain and directs it into the peripheral 

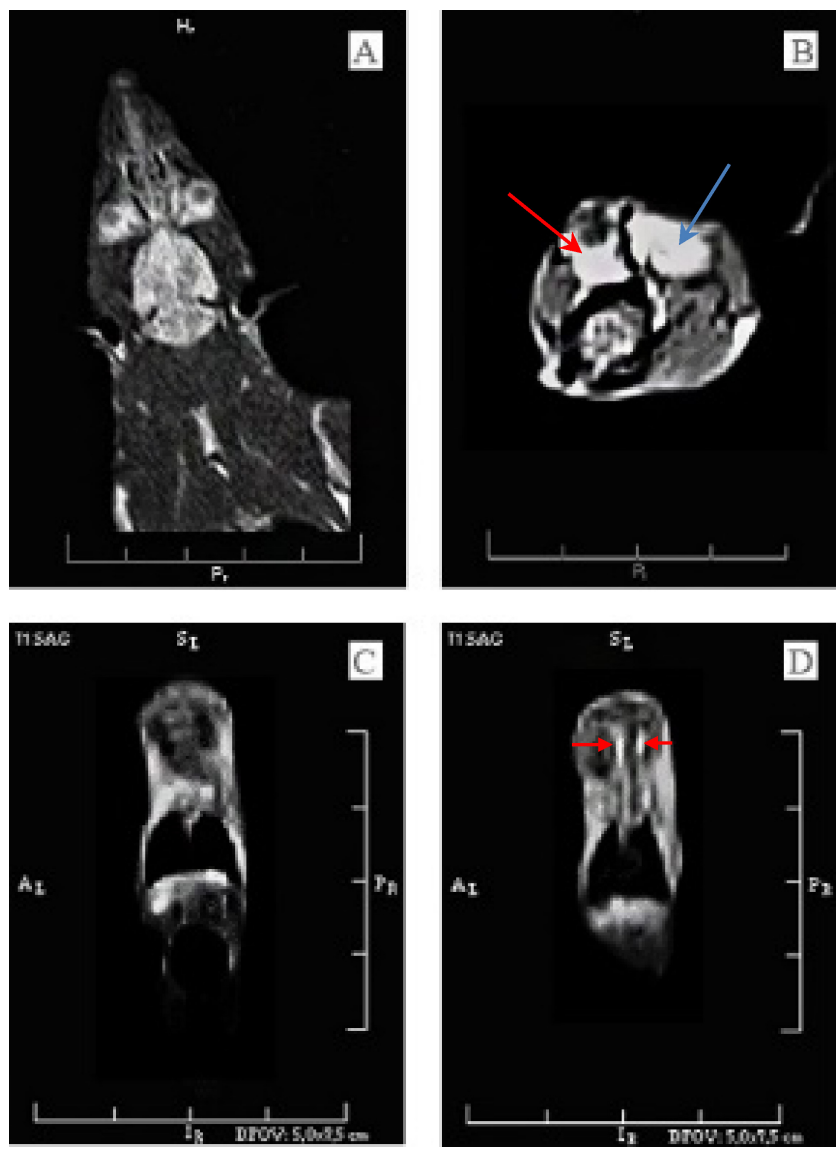

Fig. 1. The typical example of common type of stressinduced hemorrhages in the brain of adult (on the top) and newborn (on the bottom) rats: $\mathrm{A}$ and $\mathrm{C}-\mathrm{MRI}$ imaging of normal brain tissues; B and D - the intensive MRI signals show the hemorrhagic impregnation of the brain tissues; B the hemorrhages in cerebellum (blue arrow) and cerebellar nucleus (red arrow); D - small hemorrhages in the brain cortex (arrowed).
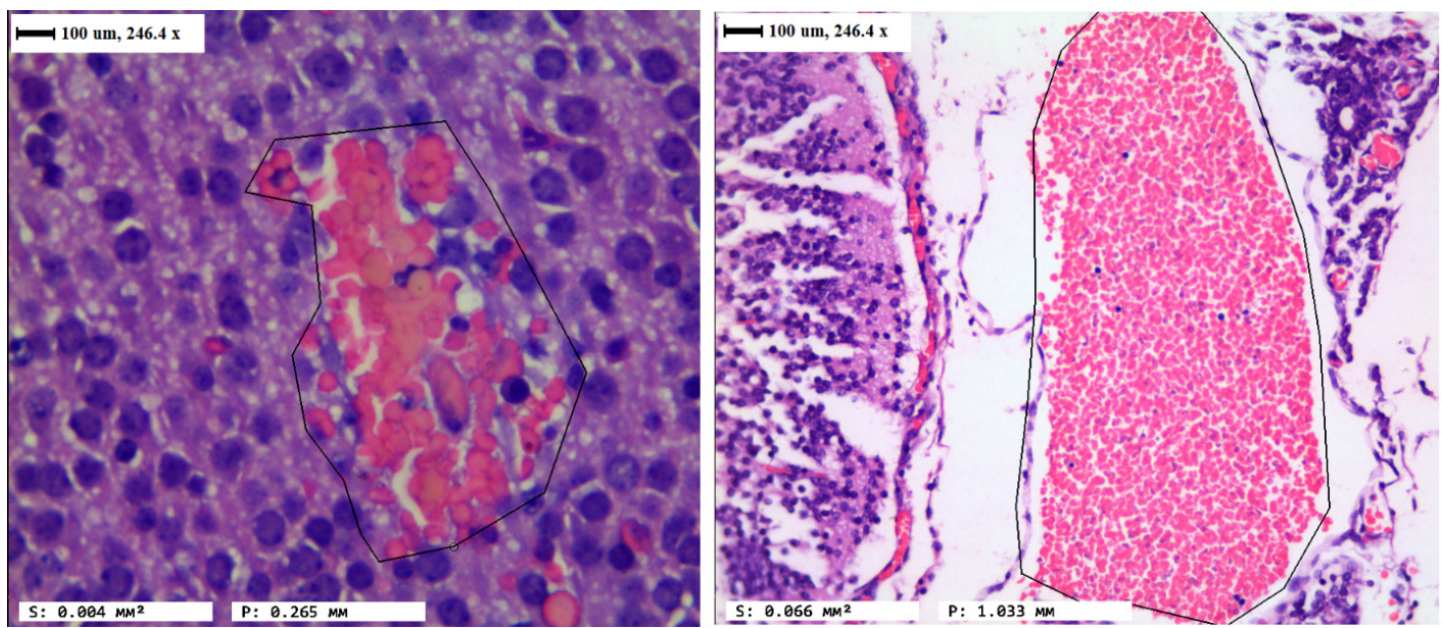

Fig. 2. The typical example of size of stress-induced hemorrhages in the brain of newborn (on the left) and adult (on the right) rats assessed by histological methods. Haematoxylin and Eosin staining. circulation. The results of this series of experiment are shown in Table 2. The basal values of hemodynamic parameters of the sagittal sinus did not differ between age groups. The development of intracranial hemorrhages was accompanied by increase in diameter of the sagittal sinus and decrease in velocity of blood flow in this vessel independently on age of rats. However, these changes were less pronounced in adult rats compared with newborn rats. So, diameter of the sagittal sinus was larger in newborn rats than in adult animals $(1.67 \pm 0.04 \mathrm{~mm}$ vs. $0.71 \pm 0.03 \mathrm{~mm}, p<0.05)$. The dilation of the sagittal sinus was accompanied by more significant decrease in blood flow velocity in newborn rats than in adult animals $(2.09 \pm 0.03 \mathrm{~mm} / \mathrm{sec}$ vs. $6.07 \pm$ $0.08 \mathrm{~mm} / \mathrm{sec}, \quad p<0.05$ for newborn rats; $3.84 \pm$ $0.07 \mathrm{~mm} / \mathrm{sec}$ vs. $6.57 \pm 0.04 \mathrm{~mm} / \mathrm{sec}, p<0.05$ for adult rats).

In the third step of our investigations, we studied age differences in the BBB permeability in normal state and after the stroke incidence using color and adrenaline tests. We used Evans Blue (dye 961Da) that becomes a high molecular weight complex $(68.5 \mathrm{kDa})$ due to strong bound to the serum albumins. Evans Blue does not permeate the BBB in normal state but can easily across the compromised BBB after brain injuries. ${ }^{26}$ The intravenous infusion of Evans Blue was not accompanied by any changes in the brain tissues in both intact newborn and adult rats. But, the stroke development was associated with the leakage of Evans Blue into the brain the next day after dye administration in adult but not in newborn rats (Fig. 3). 


\section{S.-G. Oxana et al.}

Table 2. The hemodynamic parameters of the sagittal sinus in newborn and adult rats in the control and the post-stroke groups.

\begin{tabular}{lcccccc}
\hline & \multicolumn{2}{c}{ Control groups } & & \multicolumn{2}{c}{ Post-stroke groups } \\
\cline { 2 - 3 } Parameters & Newborn rats & Adult rats & & Newborn rats & Adult rats \\
\hline Diameter of sagittal sinus, mm main trunk & $0.30 \pm 0.03$ & $0.33 \pm 0.01$ & & $1.67 \pm 0.04^{* \dagger}$ & $0.71 \pm 0.03^{*}$ \\
Left branch & $0.22 \pm 0.01$ & $0.24 \pm 0.02$ & & $0.46 \pm 0.04^{* \dagger}$ & $0.35 \pm 0.02^{*}$ \\
Right branch & $0.20 \pm 0.03$ & $0.21 \pm 0.01$ & & $0.39 \pm 0.01^{* \dagger}$ & $0.28 \pm 0.01^{*}$ \\
Blood flow velocity (in main trunk), mm/sec & $6.07 \pm 0.08$ & $6.57 \pm 0.04$ & & $2.09 \pm 0.03^{* \dagger}$ & $3.84 \pm 0.07^{*}$ \\
\hline
\end{tabular}

Note: $p<0.05$ vs.: *Basal levels; ${ }^{*}$ Age groups.

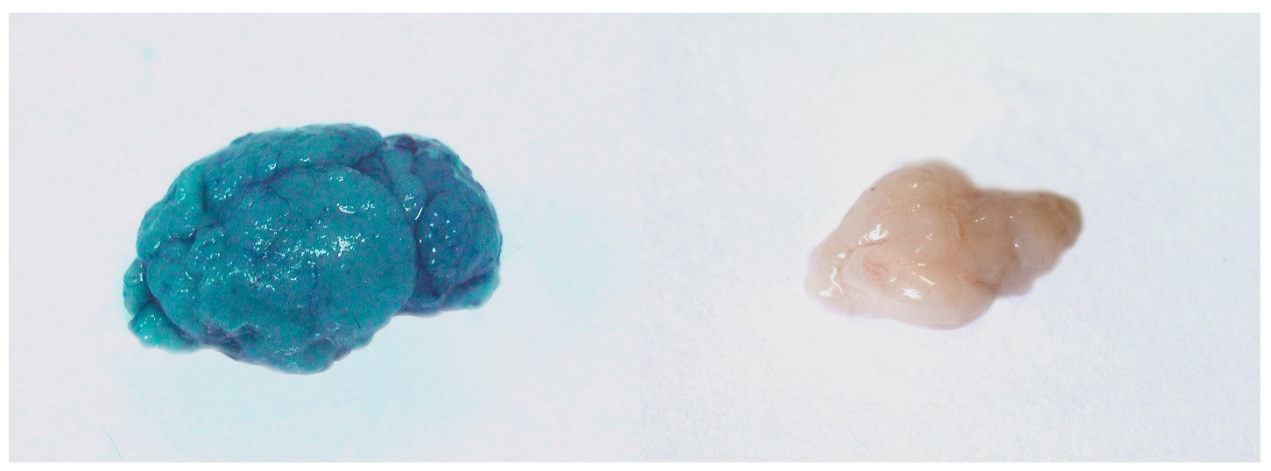

Fig. 3. The color test for the study of age differences in the stroke-induced breakdown of BBB permeability with using Evans blue injection. The leakage of Evans Blue is in adult but not in newborn rats.

The results with adrenaline test are shown in Table 3. In normal condition, adrenaline infusion caused constriction of the sagittal sinus that was accompanied by decrease in diameter of this cerebral vein. There were no age differences in vascular effect of adrenaline. So, adrenaline induced the decrease in diameter of the sagittal sinus by $30 \%$ $(p<0.05)$ in newborn rats and by $31 \%(p<0.05)$ in adult rats.

The stroke incidence was accompanied by complete loss of sensitivity of the sagittal sinus to adrenaline in newborn rats. Indeed, newborn rats with the stroke did not demonstrate any significant changes in diameter of the sagittal sinus (Fig. 4).
The adult rats demonstrated tendency to suppression of adrenergic vasoconstrictor effect. Nevertheless, they showed significant response (12\%, $p<0.05)$ to adrenaline. The changes of normal vascular responses to adrenaline during the stroke development can be explained via the difficult vessel constriction due to the critical dilation of the sagittal sinus and accumulation of extensive blood in this vessel that was more pronounced in newborn than in adult animals. Other possible mechanism may lead in age differences in the BBB permeability.

In the fourth step of our study, we studied the expression of two main structural and functional

Table 3. The changes in diameter of the sagittal sinus after adrenaline injection before and after stroke in newborn and adult rats.

\begin{tabular}{lccccc}
\hline & \multicolumn{2}{c}{ Before stroke } & & \multicolumn{2}{c}{ After stroke } \\
\cline { 2 - 3 } \cline { 5 - 6 } Groups & Basal values & Effect of adrenaline & & Basal values & Effect of adrenaline \\
\hline Newborn rats & $0.30 \pm 0.03$ & $0.21 \pm 0.02^{*}$ & & $1.67 \pm 0.04 \dagger$ & $1.65 \pm 0.07^{\dagger}$ \\
Adult rats & $0.33 \pm 0.01$ & $0.23 \pm 0.03^{*}$ & & $0.71 \pm 0.03$ & $0.63 \pm 0.05^{*}$ \\
\hline
\end{tabular}

Note: $p<0.05$ vs.: ${ }^{*}$ Basal levels; ${ }^{\dagger}$ Age groups. 


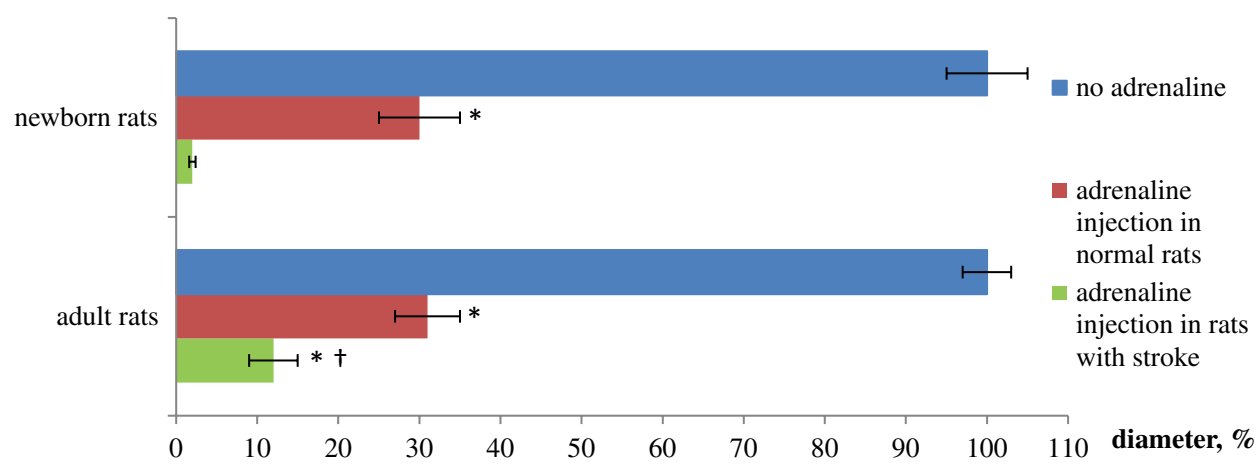

Fig. 4. The changes in diameter of the sagittal sinus (\%) after adrenaline injection in newborn and adult rats under normal state and during stroke development. The basal values of diameter of sagittal sinus (no adrenaline) were expressed as $100 \%$.

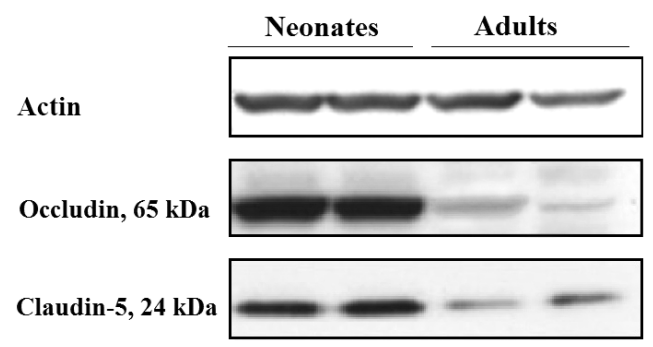

Fig. 5. Western blots showing the expression of tight junction proteins (occludin and claudin-5) in the brain of newborn and adult rats.

elements of the BBB - occludin and claudin-5 in newborn and adult rats using blotting analysis and immunohistochemistry. Figure 5 shows that the expression of both tight junction proteins was



Fig. 6. The immunohistochemistry study of expression of occludin and claudin-5 (brown color) in cerebral vessels (arrowed) in adult (on the left) and newborn (on the right) rats.
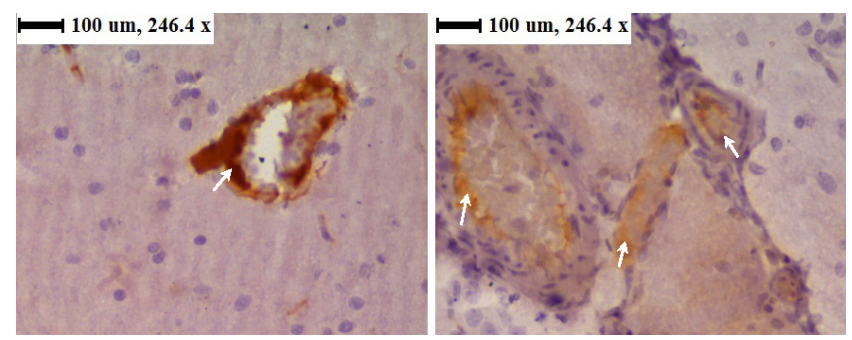

Fig. 7. The immunohistochemistry study of expression of claudin-5 (brown color) in cerebral veins (arrowed) in newborn (on the left) and adult (on the right) rats.

greater in newborn animals compared with adult rats.

The western blotting results were confirmed by immunohistochemistry data (Fig. 6). The expression of occludin and claudin-5 in the cerebral vessels were higher in newborn rats compared with adult animals. We also found the great expression of claudin-5 in large cerebral veins in newborn animals than in adult rats (Fig. 7).

\section{Discussion}

In this study, we examined age differences in the stress-related hemorrhagic stroke and mechanisms underlying these physiological processes focusing on the $\mathrm{CBF}$ and the BBB. Using histological method as a gold standard for the morphological evaluation of pathological changes in tissues, we found that sound stress induced more severe hemorrhages in adult rats compared with newborn rats. Indeed, the size of hemorrhages was larger in adult rats compared with newborn rats. MRI results showed age particularities of localization of hemorrhages in the brain. All newborn rats demonstrated small hemorrhages in 
the cortex, while in adult rats hemorrhages occurred in the cerebellum and cerebellar nucleus, i.e., in more deep areas of the brain than in newborn rats. Our results are consistent with clinical data suggesting important differences in the hemorrhagic stroke (topography, outcomes) in children when compared with adults. ${ }^{1-3}$

The cerebral venous disturbances are one of the important mechanism underlying pathological changes in the CBF associated with the stroke. ${ }^{23-25}$ It is also known that neonatal stroke is primary the venous infarction ${ }^{30,31}$ due to a weakness in the wall of cerebral veins in neonates. ${ }^{32}$ Taking into account of these facts, we analyzed the changes in hemodynamic parameters of the sagittal sinus as a main venous sinus of the brain collecting blood from the all cerebral veins. Using DOCT, we found that the stroke incidence is accompanied by relaxation of the sagittal sinus with the fall of velocity blood flow that was more pronounced in newborn rats compared with adult ones. Indeed, in newborn rats the size of the sagittal sinus increased in 2.3-fold higher and the velocity blood flow decreased in 1.8-fold higher than in adult rats. The more significant changes in a main cerebral vein in newborn vs. adult rats suggest that the stress-reactivity of venous system is greater in the neonatal vs. mature brain.

Sympathetic vasopressors such as adrenaline are commonly used in clinical practice to correct cerebral perfusion after stroke and traumatic brain injury. ${ }^{27-29}$ Here, we analyzed the effects of adrenaline on cerebral venous circulation in newborn and adult rats in normal state and after stroke development. Our results showed that adrenaline caused the constrictor effect on the sagittal sinus in healthy animals without any age differences. However, the stroke is associated with complete loss of sensitivity of the sagittal sinus to adrenaline in newborn rats while adult rats demonstrated weak but statistically significant vasoconstrictor response. The relaxation of the sagittal sinus is a sign of accumulation of excessive blood in cerebral venous system that makes difficult vessel constriction to adrenergic stimulation. The more pronounced relaxation of the sagittal sinus in newborn vs. adult rats partly explains the total loss of adrenaline effects on the sagittal sinus in the first case. Other possible mechanism underlying age differences in adrenaline vascular effects can be the different changes in the BBB permeability in newborn and adult animals under normal state and during stroke development.
Using standard color test, we performed intravenous injection of Evans Blue, across the intact $\mathrm{BBB}$ but it can permeate the injured BBB. ${ }^{26} \mathrm{We}$ found that Evans Blue permeated the stroke-injured BBB in adult rats but not in newborn animals. This fact suggests that the stroke causes the increase in the BBB permeability in the mature brain but not in neonatal brain in rats. Other researchers using model of ischemic stroke have showed the similar results. ${ }^{13}$

Using western blotting and immunochemistry study of expression of occludin and claudin-5, we found that more higher resistance of the $\mathrm{BBB}$ to stroke-injures in newborn vs. adult rats was associated with the greater expression of these two main tight junction proteins of the BBB in neonatal vs. mature brain of animals.

Collectively, our results show age differences in the stroke development and possible mechanisms underlying these processes. The same factor as severe sound stress induces different hemorrhages in newborn and adult rats: small superficial bleedings in the cortex in newborn rats and more severe deep bleedings in the cerebellum in the adult rats. The cerebral venous circulatory system is more sensitive to harmful effect of stress in newborn rats than in adult animals. Despite this fact, stressed adult but not newborn rats demonstrated the breakdown of BBB permeability. The one of possible mechanisms underlying the higher resistance to stress-related stroke injures of cerebral vessels in newborn rats compared with adult animals is the greater expression of two main tight junction proteins of $\mathrm{BBB}$ (occludin and claudin-5) in neonatal vs. mature brain in rats.

\section{Acknowledgment}

This work was supported by Grant of Russian Science Foundation (No. 14-15-00128).

\section{References}

1. J. K. Lynch, "Epidemiology and classification of perinatal stroke," Semin. Fetal. Neonatal. Med. Rev. 14, 245-249 (2009).

2. W. Lo, "Childhood hemorrhagic stroke: An important but understudied problem," J. Child. Neurol. Rev. 26, 1174-1185 (2011).

3. H. J. Fullerton, Y. W. Wu, S. Zhao, S. C. Johnston, "Risk of stroke in children: Ethnic and gender disparities," Neurology. Rev. 61, 189-194 (2003). 
4. J. Broderick, G. T. Talbot, E. Prenger, A. Leach, T. Brott, "Stroke in children within a major metropolitan area: The surprising importance of intracerebral hemorrhage," J. Child. Neurol. Rev. 8, 250-255 (1993).

5. M. Giroud, M. Lemesle, J. B. Gouyon et al., "Cerebrovascular disease in children under 16 years of age in the city of Dijon, France: A study of incidence and clinical features from 1985 to 1993," J. Clin. Epidemiol. Rev. 48, 1343-1348 (1995).

6. H. J. Fullerton, Y. W. Wu, S. Sidney, S. C. Johnston, "Recurrent hemorrhagic stroke in children: A population-based cohort study," Stroke. Rev. 38, 2658-2662 (2007).

7. H. J. Fullerton, Y. W. Wu, S. Sidney, S. C. Johnston, "Risk of recurrent childhood arterial ischemic stroke in a population-based cohort: The importance of cerebrovascular imaging," Pediatri. Rev. 119, 495-501 (2007).

8. P. M. Rothwell, A. J. Coull, M. F. Giles et al., "Change in stroke incidence, mortality, case-fatality, severity, and risk factors in Oxfordshire, UK from 1981 to 2004 (Oxford Vascular Study)," Lancet. Rev. 363, 1925-1933 (2004).

9. A. I. Qureshi, M. F. Suri, A. Nasar et al., "Changes in cost and outcome among US patients with stroke hospitalized in 1990 to 1991 and those hospitalized in 2000 to 2001," Stroke. Rev. 38, 2180-2184 (2007).

10. V. L. Feigin, C. M. Lawes, D. A. Bennett, C. S. Anderson, "Stroke epidemiology: A review of populationbased studies of incidence, prevalence, and case-fatality in the late 20th century," Lancet Neurol. Rev. 2, 43-53 (2003).

11. D. Della-Morte, F. Guadagni, R. Palmirotta, G. Testa et al., "Genetics of ischemic stroke, strokerelated risk factors, stroke precursors and treatments," Pharmacogenomics. Rev. 13(5), 595613 (2012).

12. J. Wang, A. J. Licht, "Pediatric perfusion MR imaging using arterial spin labeling," Neuroimag. Clin. N. Am. Rev. 16, 149-167 (2006).

13. D. Fernández-López, J. Faustino, R. Daneman, L. Zhou, S. Y. Lee, N. Derugin et al., "Blood-brain barrier permeability is increased after acute adult stroke but not neonatal stroke in the rat," J. Neurosc. Rev. 32(28), 9588-9600 (2012).

14. J. K. Lynch, D. G. Hirtz, G. DeVeber, K. B. Nelson, "Report of the national institute of neurological disorders and stroke workshop on perinatal and childhood stroke," Pediatri. Rev. 109, 116-123 (2002).

15. N. R. Saunders, M. D. Habgood, K. M. Dziegielewska, "Barrier mechanisms in the brain, II. Immature brain," Clin. Exp. Pharmacol. Physiol. Rev. 26, 85-91 (1999).
16. Ek. CJ, Wong, S. A. Liddelow, P. A. Johansson, K. M. Dziegielewska, N. R. Saunders, "Efflux mechanisms at the developing brain barriers: ABCtransporters in the fetal and postnatal rat," Toxicol. Lett. Rev. 197, 51-59 (2010).

17. R. Daneman, L. Zhou, A. A. Kebede, B. A. Barres, "Pericytes are required for blood-brain barrier integrity during embryogenesis," Nature Rev. 468, 562-566 (2010b).

18. L. C. Jordan, A. E. Hillis, "Hemorrhagic Stroke in Children," Pediatr. Neurol. Rev. 36(2), 73-80 (2007).

19. J. K. Lynch, C. J. Han, "Pediatric stroke: What do we know and what do we need to know?,"Semin. Neurol. Rev. 25, 410-423 (2005).

20. J. P. Broderick, H. P. Adams, W. Barsan, W. Feinberg, E. Feldmann, J. Grotta et al., "Guidelines for the management of spontaneous intracerebral hemorrhage: A statement for healthcare professionals from a special writing group of the Stroke Council, American Heart Association," Stroke. Rev. 30, 905-915 (1999).

21. Committee for the Update of the Guide for the Care and Use of Laboratory Animals, Institute for Laboratory Animal Research, Division on Earth and Life Studies, National Research Council of the National Academies. Guide for the care and use of laboratory animals. 8th Edition. Washington: The National Academies Press, 2011. Available at http://oacu.od.nih.gov/regs/guide/guide.pdf.

22. T. Romanova, "The method of modeling of cerebral hematoma," Patol. Fiziol. Rev. 3, 80-91 (1989).

23. A. N. Pavlov, O. V. Semyachkina-Glushkovskaya, Y. Zhang, O. A. Bibikova, O. N. Pavlova, Q. Huang, D. Zhu, P. Li, V. V. Tuchin, Q. Luo, "Multiresolution analysis of pathological changes in cerebral venous dynamics in newborn mice with intracranial hemorrhage: adrenorelated vasorelaxation," Physiol. Meas. Rev. 35, 1983-1999 (2014).

24. O. V. Semyachkina-Glushkovskaya, V. V. Lychagov, O. A. Bibikova, I. A. Semyachkin-Glushkovskiy, S. S. Sindeev, E. M. Zinchenko, M. M. Kassim, H. A. Braun, F. Al-Fatle, L. Al Hassani, V. V. Tuchin, "The assessment of pathological changes in cerebral blood flow in hypertensive rats with stressinduced intracranial hemorrhage using Doppler OCT: particularities of arterial and venous alterations," J. Photonics Lasers Med. Rev. 2(2), 109-116 (2013).

25. O. Semyachkina-Glushkovskaya, V. Lychagov, O. Bibikova, I. Semyachkin-Glushkovskiy, S. Sindeev, E. Zinchenko, M. Kassim, H. Braun, F. Al-Fatle, L. Al Hassani, V. Tuchin, "The experimental study of stress-related pathological changes in cerebral venous blood flow in newborn rats assessed by 
DOCT," J. Innov. Opt. Health Sci. Rev. 3(3), 1350023 (10 pages) (2013).

26. L. Belayev, R. Busto, W. Zhao, M. D. Ginsberg, "Quantitative evaluation of blood-brain barrier permeability following middle cerebral artery occlusion in rats," Brain Res. Rev. 739, 88-96 (1996).

27. J. L. Di Gennaro, D. C. Mack, A. Malakouti, J. J. Zimmerman, W. Armstead, M. S. Vavilala, "Use and effect of vasopressors after pediatric traumatic brain injury," Dev. Neurosci. Rev. 32(5-6), 420-430 (2011).

28. P. Sookplung, A. Siriussawakul, A. Malakouti, D. Sharma, J. Wang, M. Souter, R. Chesnut, M. Vavilala, "Vasopressor use and effect on blood pressure after severe adult traumatic brain injury," Neurocrit. Care. Rev. 15(1), 46-54 (2011).
29. A. Azhan, F. Y. Wong, "Challenges in understanding the impact of blood pressure management on cerebral oxygenation in the preterm brain," Front. Physiol. Rev. 3, 3-8 (2012).

30. H. S. Ghazi-Birry, W. R. Brown, D. M. Moody, V. R. Challa, S. M. Block, D. M. Reboussin, "Human germinal matrix: Venous origin of hemorrhage and vascular characteristics," AJNR Am. J. Neuroradiol. Rev. 18, 219-229 (1997).

31. C. J. Bruno, L. A. Beslow, C. M. Witmer et al., "Haemorrhagic stroke in term and late preterm neonates," Arch. Dis. Child Fetal Neonatal Ed. 99, F48-F53 (2014).

32. G. Hambleton, J. S. Wigglesworth, "Origin of intraventricular hemorrhage in the preterm infant," Arch. Dis. Child. Rev. 51, 651-659 (1976). 